\title{
Revisiting a detached stellar structure in the outer northeastern region of the Small Magellanic Cloud
}

\author{
Andrés E. Piatti ${ }^{1,2 \star}$ \\ ${ }^{1}$ Instituto Interdisciplinario de Ciencias Básicas (ICB), CONICET-UNCUYO, Padre J. Contreras 1300, M5502JMA, Mendoza, Argentina \\ ${ }^{2}$ Consejo Nacional de Investigaciones Científicas y Técnicas, Godoy Cruz 2290, C1425FQB, Buenos Aires, Argentina
}

Accepted XXX. Received YYY; in original form ZZZ

\begin{abstract}
The outer northeastern region of the Small Magellanic Cloud (SMC) is populated by a shell-like overdensity whose nature was recently investigated. We analyzed twenty catalogued star clusters projected onto it from Survey of the MAgellanic Stellar History data sets. After carrying out a cleaning of field stars in the star cluster colourmagnitude diagrams (CMDs), and deriving their astrophysical properties from the comparison between the observed and synthetic CMDs, we found that four objects are not genuine star clusters, while the remaining ones are young star clusters (11, age $\sim 30-200 \mathrm{Myr})$ and intermediate-age (5, age $~ 1.7-2.8$ Gyr) star clusters, respectively. The resulting distances show that intermediate-age and some young star clusters belong to the SMC main body, while the remaining young star clusters are nearly 13.0 $\mathrm{kpc}$ far away from those in the SMC, revealing that the shell-like overdensity is more extended along the line-of-sight than previously thought. We also found a clear age trend and a blurred metallicity correlation along the line-of-sight of young clusters, in the sense that the farther a star cluster from the SMC, the younger, the more metal rich, and the less massive it is. These young clusters are also affected by a slightly larger interstellar reddening than the older ones in the shell-like overdensity. These outcomes suggest that the shell-like overdensity can possibly be another tidally perturbed/formed SMC stellar structure from gas striped off its body, caused by the interaction with the Large Magellanic Cloud or the Milky Way.
\end{abstract}

Key words: galaxies: individual: SMC - galaxies: star clusters: general - methods: observational

\section{INTRODUCTION}

Martínez-Delgado et al. (2019) studied the nature of a stellar structure located toward the outer northeastern region of the Small Magellanic Cloud (SMC), which they referred to as a shell-like overdensity. By using the Survey of the MAgellanic Stellar History (SMASH, Nidever et al. 2017) and the second release (DR2) of the Gaia mission (Gaia Collaboration et al. 2016, 2018) data sets, they inferred that the shell-like overdensity is composed by stars younger than $\sim$ $150 \mathrm{Myr}$. As far as its origin is considered, they concluded that it formed from a recent star formation even, possible caused by the interaction of the SMC with the Large Magellanic Cloud (LMC) or the Milky Way (MW), and discarded the possibilities of being a tidally disrupted stellar system or a bright part of a spiral arm-like structure. From available

* E-mail: andres.piatti@unc.edu.ar ages of nine star clusters projected onto the shell-like overdensity, they confirmed the youth of such a stellar structure.

The resulting stellar content and structure of the shell-like overdensity are based on the analysis of colourmagnitude diagrams (CMDs), assuming a mean distance modulus of $18.96 \mathrm{mag}$. The same fixed distance modulus was also assumed for the nine studied star clusters. However, the SMC is known to be more extended along the line-of-sight than the size of the galaxy projected in the sky (Ripepi et al. 2017; Muraveva et al. 2018; Graczyk et al. 2020), so that by adopting a mean distance for every single star and star cluster could mislead the interpretation about the structure, stellar population and the possible origin of this SMC stellar structure. As far as we are aware, accurate distances of star clusters have been previously measured for a limited number of objects (19 star clusters; Crowl et al. 2001; Glatt et al. 2008; Dias et al. 2016; Martínez-Vázquez et al. 2021; Dias et al. 2021). Recently, Piatti (2021b) considered the SMC as a triaxial spheroid, and estimated deprojected distances for 
the SMC star clusters catalogued by Bica et al. (2020). By adopting a 3D geometry of the SMC, he avoided the spurious effects caused by assuming that a star cluster observed along the line-of-sight is close to the galactic centre.

Precisely, the main aim of this work is to provide for the first time accurate distances, ages and metallicities for all the star clusters projected onto the shell-like overdensity, thus mitigating the lack of homogeneous estimates of these astrophysical properties. Fortunately, this scope is nowadays possible to be achieved because of the availability of fairly deep photometry and sophisticated statistical methods to fit theoretical isochrones to the star clusters' CMDs. An additional and not negligible aspect of this analysis comprises the appropriate decontamination of field stars from the star clusters' CMDs. The combined star cluster CMD built by Martínez-Delgado et al. (2019, see their figure 8) shows mainly the contribution of main sequence stars of massive young star clusters down to $i \sim 22 \mathrm{mag}$ and of fainter stars which belong to the composite SMC star field. Both cluster and field stars may populate the observed combined subgiant, red giant branches and red clumps, so that intermediate-age and young clusters should not be ruled out. For this reason, a field star cleaning of the observed star clusters' CMDs is necessary before estimating their ages, distances and metallicities.

Once cleaned star clusters' CMDs are obtained, it is possible to investigate their 3D spatial distribution, and hence to draw conclusions about the location and dimensions of the shell-like overdensity, provided that they belong to that stellar structure. Their spatial distribution can also hint at its possible origin, namely, as a stellar structure that formed recently in the outskirts of the SMC, a detachment of some part of the SMC, or both mechanisms combined. Bearing in mind the above considerations, we describe in Section 2 the gathered star cluster sample, the publicly available retrieved data sets, and the cleaning procedure applied in order to build star clusters' CMDs with highly probable members. Section 3 deals with the cleaned CMDs and cluster properties estimates, while in Section 4 we analyze and discuss the nature of the shell-like overdensity. The main conclusions of this work are given in Section 5.

\section{DATA HANDLING}

We started by searching the star cluster catalogue compiled by Bica et al. (2020) any star cluster located within the region of the shell-like overdensity delimited by MartínezDelgado et al. (2019). Particularly, we used as reference the boundaries of their figure 8 (upper panel), which are also illustrated in Fig. 1. We found 20 catalogued star clusters, among them the nine star clusters used by Martínez-Delgado et al. (2019, see their Table 1). Four catalogued star clusters (OGLE-SMC 274, 275, 276, and 276) were not confirmed as genuine physical systems in our field star decontamination procedure, so that we discarded them from our analysis. The remaining catalogued star clusters are listed in Table 1.

We used the Survey of the Magellanic Stellar History (SMASH) DR2 data sets (Nidever et al. 2021) to conduct the analysis of the selected star clusters. Coordinates (R.A. and Dec.), PSF $g, i$ magnitudes and their respective errors, $\chi$ and SHARPNESS parameters of stellar sources located inside

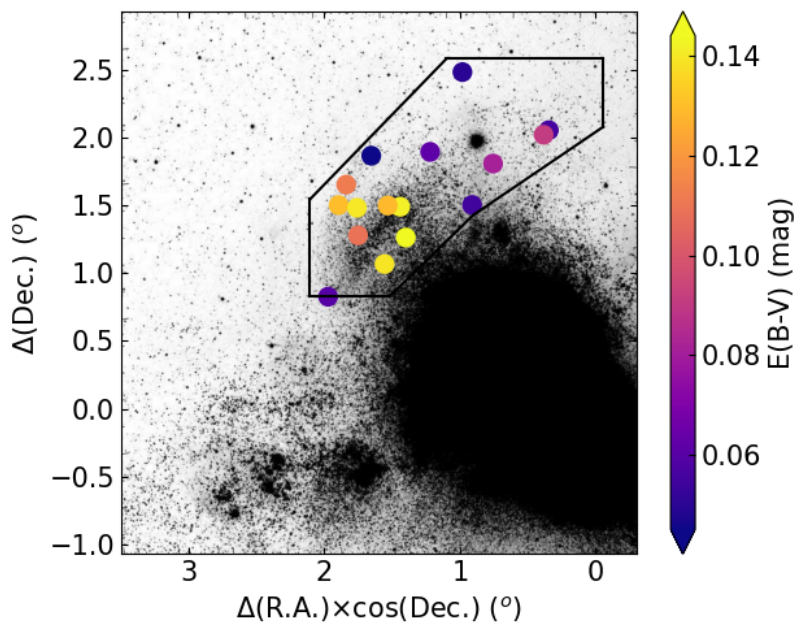

Figure 1. Coloured symbols represent star clusters located within the shell-like overdensity as delimited by Martínez-Delgado et al. (2019, Fig. 8), overplotted over their Fig. 5.

a radius of $9^{\prime}$ from the star clusters' centres listed by Bica et al. (2020) were retrieved from the portal of the Astro Data $\mathrm{Lab}^{1}$, which is part of the Community Science and Data Center of NSF's National Optical Infrared Astronomy Research Laboratory. The retrieved data sets consist of sources with $0.2 \leq$ SHARPNESS $\leq 1.0$ and $\chi^{2}<0.5$, so bad pixels, cosmic rays, galaxies, and unrecognized double stars were excluded. According to Bica et al. (2020), the radii of the selected star clusters are relatively small, from $0.35^{\prime}$ up to $1.7^{\prime}$, with an average of $0.9^{\prime}$ (NGC 458 is the largest cluster in the sample with a radius of $2.6^{\prime}$ ). Because we downloaded information for circular areas much larger than the star clusters' radii, we thoroughly monitored the contamination of field stars in the star clusters' CMDs.

When dealing with star cluster CMDs, it is necessary to take into account the contamination of field stars, because it is not straightforward to consider a star as a cluster member only on the basis of its position in that CMD. Frequently it is assumed that the colours and magnitudes of field stars projected onto the star cluster field are similar to those around the star cluster. However, even though a star cluster is not projected onto a crowded star field or is not affected by differential reddening, it is highly possible to find different magnitude and colour distributions of field stars located along the star cluster line-of-sight, and in the surrounding field as well. For this reason, it is convenient to use field stars located all around the star cluster.

The field star decontamination procedure adopted in this work is based on that devised by Piatti \& Bica (2012), which was satisfactorily applied in cleaning CMDs of star clusters projected toward crowded star fields (e.g., Piatti 2017, and references therein) and affected by differential reddening (e.g., Piatti 2018, and references therein). We refer the reader to the recent works by Piatti (2021a) and Piatti (2021c), where details of the cleaning technique are provided. The method comprises three main steps: 1) to properly reproduce the distribution of magnitudes and colours of

1 https://datalab.noao.edu/smash/smash.php 
stars in the surroundings of the star cluster; 2) to reliably subtract those magnitude and colour distributions from the star cluster CMD and; 3) to assign membership probabilities to stars that survived the cleaning procedure. Stars with relatively high membership probabilities can likely be cluster members, provided that they are placed along the expected CMD star cluster sequences and belong to a stellar overdensity.

The sky regions used to map the field star density, and the distribution of magnitudes and colours of field stars consist of six circles of radius $3^{\prime}$ distributed uniformly around the star cluster circle, which is also of the same radius. These surrounding circles are thought to be placed far from the star cluster, but not too far from it as to become unsuitable as representative of the star field projected along the line-ofsight of the star cluster. We cleaned a star cluster CMD six times. Each execution used the observed star cluster CMD and one of the six associated surrounding fields, and produced a cleaned star cluster CMD. Thus, we obtained six different cleaned CMDs for each star cluster. During each individual run, we subtracted from a star cluster CMD a number of stars equal to that in the chosen surrounding field. In order to subtract stars with the same magnitude and colour distributions of the field stars, we defined boxes centred on the magnitude and colour of each star of the surrounding field CMD, then superimposed them on the star cluster CMD, and finally chose one star per box to subtract. In the present work, we used initial boxes of $(\Delta g, \Delta(g-i))$ $=(1.0 \mathrm{mag}, 0.25 \mathrm{mag})$ centred on the $(g, g-i)$ values of each field star. In the case that more than one star is located inside that delimited CMD region, the closest one to the centre of that (magnitude, colour) box is subtracted.

We finally used the six different cleaned star cluster CMDs to assign membership probabilities to surviving stars, as follows: $P(\%)=100 \times N / 6$, where $N$ represents the number of times a star was not subtracted during the six different CMD cleaning executions. Hence, a star with $P=100$ is a star not subtracted during any of the cleaning runs that involved six different surrounding fields, separately. For this reason, it has the highest chance to contribute to the intrinsic features of the cleaned CMD. A star with $\mathrm{P}=16.67$ is that that survived once from six different cleaning executions, meaning that its magnitude and colour were mostly found in the surrounding field population. With that information on hand, we built Fig. 2, which shows the spatial distribution and the CMD of all the measured stars of the selected star cluster sample.

\section{STAR CLUSTER PARAMETERS}

The resulting cleaned CMDs highlight the star cluster sequences on top of those of field stars. As can be seen, there seem to be moderately old star clusters projected onto younger star fields (e.g., BS 116, L110); young clusters projected onto older fields (e.g., L95, NGC 458); young star clusters projected onto nearly young star fields (e.g., B88, H86-197); and relatively old star clusters projected onto star fields of similar age (e.g., HW 56, L73). A common feature seen in the cleaned CMDs is the presence of field stars $(P<$ $50 \%)$ at the fainter part of the Main Sequence, as expected. Because of stochastic effects some residuals at any $P$ value
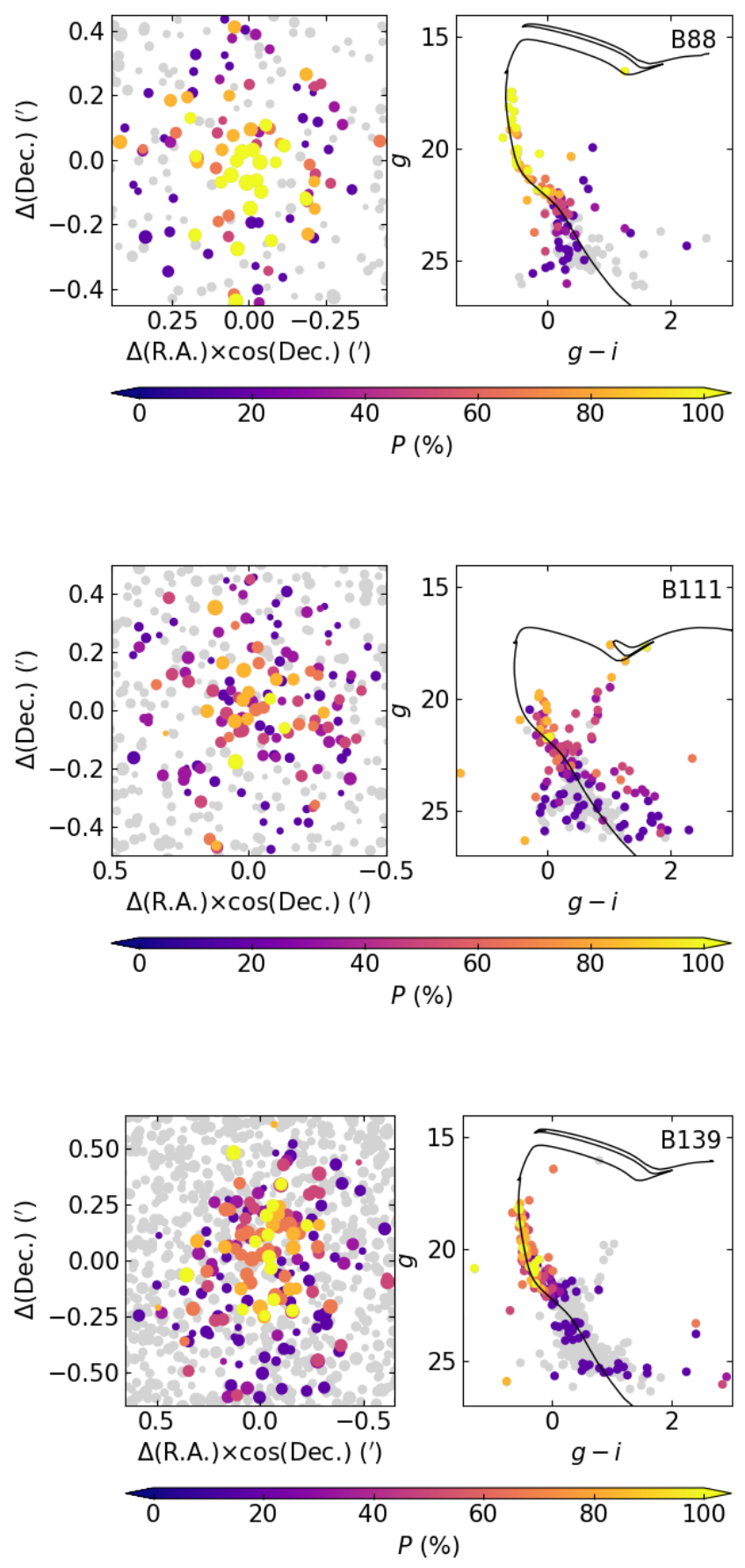

Figure 2. Charts of the star clusters (left panel), and the respective CMDs (right panel), drawn with grey symbols. The size of the charts' symbols is proportional to the $g$ brightness of the star. Coloured symbols in both panels represent the stars that survived the CMD cleaning procedure, colour-coded according to the assigned membership probabilities $(P)$. A theoretical isochrone (Bressan et al. 2012) for the mean derived parameters is superimposed onto the CMD with a black line. 
Table 1. Derived astrophysical properties of selected SMC star clusters

\begin{tabular}{|c|c|c|c|c|c|c|}
\hline Star cluster & $\begin{array}{c}\mathrm{E}(\mathrm{B}-\mathrm{V}) \\
(\mathrm{mag})\end{array}$ & $\begin{array}{c}m-M_{o} \\
(\mathrm{mag})\end{array}$ & $\log ($ age /yr) & $\begin{array}{c}{[\mathrm{Fe} / \mathrm{H}]} \\
(\mathrm{dex})\end{array}$ & $\begin{array}{l}\text { Mass } \\
\left(\mathrm{M}_{\odot}\right)\end{array}$ & binarity \\
\hline B88 & $0.056 \pm 0.030$ & $18.998 \pm 0.096$ & $8.131 \pm 0.159$ & $0.0086 \pm 0.0029$ & $622 \pm 190$ & $0.27 \pm 0.14$ \\
\hline B111 & $0.081 \pm 0.048$ & $18.868 \pm 0.153$ & $8.271 \pm 0.148$ & $0.0119 \pm 0.0022$ & $212 \pm 91$ & $0.30 \pm 0.14$ \\
\hline B139 & $0.144 \pm 0.035$ & $19.098 \pm 0.111$ & $7.812 \pm 0.035$ & $0.0076 \pm 0.0042$ & $1494 \pm 398$ & $0.32 \pm 0.12$ \\
\hline BS 116 & $0.055 \pm 0.039$ & $18.749 \pm 0.124$ & $9.274 \pm 0.070$ & $0.0026 \pm 0.0007$ & $1740 \pm 417$ & $0.29 \pm 0.13$ \\
\hline H86-197 & $0.112 \pm 0.034$ & $18.798 \pm 0.108$ & $7.966 \pm 0.440$ & $0.0106 \pm 0.0024$ & $371 \pm 171$ & $0.28 \pm 0.14$ \\
\hline HW 33 & $0.091 \pm 0.035$ & $18.956 \pm 0.111$ & $7.918 \pm 0.365$ & $0.0083 \pm 0.0041$ & $1057 \pm 311$ & $0.26 \pm 0.14$ \\
\hline HW 56 & $0.062 \pm 0.039$ & $18.749 \pm 0.124$ & $9.442 \pm 0.060$ & $0.0022 \pm 0.0008$ & $2251 \pm 722$ & $0.30 \pm 0.13$ \\
\hline HW 64 & $0.142 \pm 0.026$ & $19.193 \pm 0.083$ & $7.468 \pm 0.309$ & $0.0064 \pm 0.0029$ & $1046 \pm 288$ & $0.29 \pm 0.14$ \\
\hline HW 67 & $0.045 \pm 0.040$ & $18.709 \pm 0.127$ & $9.276 \pm 0.046$ & $0.0027 \pm 0.0013$ & $2189 \pm 563$ & $0.36 \pm 0.11$ \\
\hline HW 73 & $0.130 \pm 0.014$ & $19.180 \pm 0.045$ & $7.868 \pm 0.085$ & $0.0073 \pm 0.0037$ & $1621 \pm 512$ & $0.25 \pm 0.14$ \\
\hline IC 1655 & $0.129 \pm 0.012$ & $18.659 \pm 0.039$ & $8.054 \pm 0.107$ & $0.0037 \pm 0.0015$ & $4752 \pm 180$ & $0.17 \pm 0.06$ \\
\hline IC 1660 & $0.139 \pm 0.017$ & $18.813 \pm 0.055$ & $7.909 \pm 0.143$ & $0.0054 \pm 0.0023$ & $3960 \pm 497$ & $0.15 \pm 0.09$ \\
\hline L73 & $0.050 \pm 0.041$ & $18.829 \pm 0.131$ & $9.269 \pm 0.036$ & $0.0034 \pm 0.0010$ & $3716 \pm 676$ & $0.27 \pm 0.11$ \\
\hline L95 & $0.140 \pm 0.030$ & $19.176 \pm 0.096$ & $7.585 \pm 0.372$ & $0.0067 \pm 0.0041$ & $910 \pm 326$ & $0.24 \pm 0.15$ \\
\hline L100 & $0.060 \pm 0.031$ & $18.781 \pm 0.099$ & $9.417 \pm 0.049$ & $0.0035 \pm 0.0005$ & $4649 \pm 272$ & $0.11 \pm 0.07$ \\
\hline NGC 458 & $0.109 \pm 0.010$ & $18.736 \pm 0.033$ & $8.130 \pm 0.070$ & $0.0062 \pm 0.0011$ & $4853 \pm 126$ & $0.06 \pm 0.04$ \\
\hline
\end{tabular}

also remain, which confirm that the assumption of a uniform surrounding star field is not appropriate.

We used the cleaned star cluster CMDs to derive accurate star cluster parameters by employing routines of the Automated Stellar Cluster Analysis code (ASteCA, Perren et al. 2015) that allowed us to derive all of them simultaneously. ASteCA is a suit of tools designed to analyze data sets of star clusters in order to determine their basic properties. We thus obtained a synthetic CMD that best matches the cleaned star cluster CMD. The metallicity, age, distance, reddening, star cluster present mass and binary fraction associated to that generated synthetic CMD were adopted as the best-fitted star cluster properties.

We started by using the theoretical isochrones computed by Bressan et al. (2012) for the SMASH photometric system. We downloaded theoretical isochrones for different metallicities values, from $Z=0.000152([\mathrm{Fe} / \mathrm{H}]=-2.0 \mathrm{dex})$ up to $0.030152([\mathrm{Fe} / \mathrm{H}]=0.30 \mathrm{dex})$ in steps of $\Delta Z=0.001$. This metallicity range covers almost all the metallicity regime of the Magellanic Clouds (Piatti \& Geisler 2013). This is an important consideration, because the studied star clusters lie in an outer region of the SMC, where metal-poor old and metal-rich young objects formed at the galaxy formation and galaxy interaction, respectively. As for ages, we downloaded isochrones from $\log ($ age $/ \mathrm{yr})=6.0(1 \mathrm{Myr})$ up to 10.1 (12.5 Gyr $)$ in steps of $\Delta \log ($ age $/ y r)=0.025$. In total, we gathered nearly 7800 different theoretical isochrones.

The steps by which a synthetic star cluster for a given set of age, metallicity, distance modulus, and reddening values is generated by ASteCA is as follows: i) a theoretical isochrone is picked up, densely interpolated to contain a thousand points throughout its entire length, including the most evolved stellar phases. ii) The isochrone is shifted in colour and magnitude according to the $E(B-V)$ and $(m-M)_{o}$ values to emulate the effects these parameters have over the isochrone in the CMD. iii) The isochrone is trimmed down to a certain faintest magnitude according to the limiting magnitude derived from the CMD. iv) An initial mass function (IMF) is sampled in the mass range [ 0.01-100] $M_{\odot}$ up to a total mass value $M$ provided that evolved CMD regions result properly populated. The distribution of masses is then used to obtain a properly populated synthetic CMD by keeping one star in the interpolated isochrone for each mass value in the distribution. v) A random fraction of stars is assumed to be binaries, which is set by default to $50 \%$ (von Hippel 2005), with secondary masses drawn from a uniform distribution between the mass of the primary star and a fraction of it given by a mass ratio parameter set to 0.5. vi) An appropriate magnitude completeness and an exponential photometric error functions are finally applied to the synthetic star cluster.

The input data sets consist of the cleaned $g, i$ photometry with their respective uncertainties, alongside the associated membership probabilities, i.e., all coloured points in Fig. 2. For generating the synthetic CMDs, we adopted the initial mass function of Kroupa (2002); a minimum mass ratio for the generation of binaries of 0.5 ; and a range of true distance moduli from $18.5 \mathrm{mag}(50 \mathrm{kpc})$ up to $19.5 \mathrm{mag}$ (80 kpc). Star cluster mass and binary fractions were set in the ranges $100-5000 \mathrm{M}_{\odot}$ and 0.0-0.5, respectively. We explored the parameter space of the synthetic CMDs through the minimization of the likelihood function defined by Tremmel et al. (2013, ; the Poisson likelihood ratio (eq. 10)) using a parallel tempering Bayesian MCMC algorithm, and the optimal binning Knuth (2018)'s method. Errors in the obtained parameters are estimated from the standard bootstrap method described in Efron (1982). We refer the reader to the work of Perren et al. (2015) for details concerning the implementation of these algorithms. Table 1 lists the resulting parameters for the studied star cluster sample. We illustrate the performance of the parameter matching procedure by superimposing the isochrone corresponding to the best-fitted synthetic CMD to the cleaned star cluster CMDs (see Fig. 2).

\section{ANALYSIS AND DISCUSSION}

By inspecting Table 1 we found that more than $30 \%$ of the star clusters turned out to be older than $1.7 \mathrm{Gyr}$, while the remaining ones resulted to be younger than $150 \mathrm{Myr}$. 

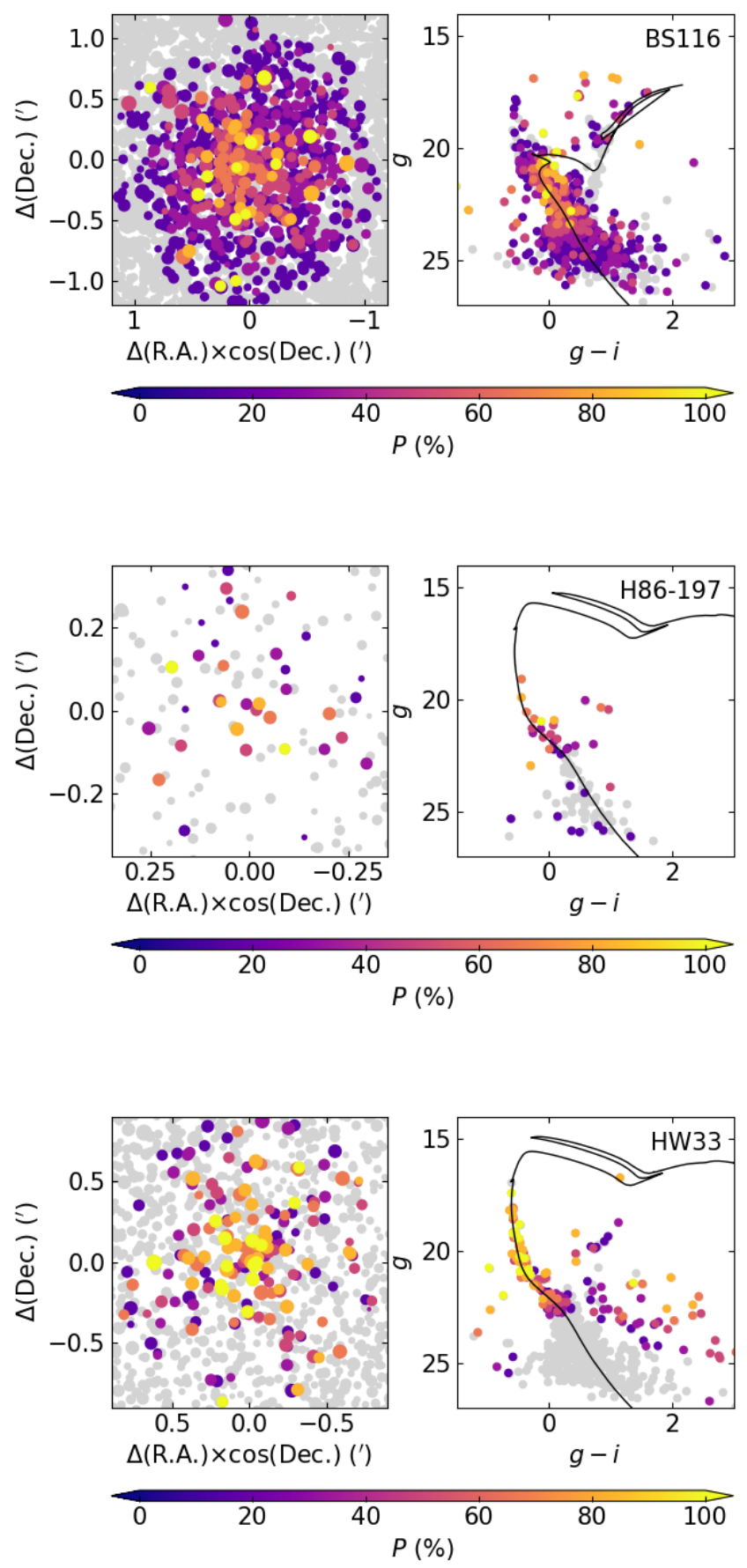

Figure 2. continued.

We confirmed that the nine star clusters used by MartínezDelgado et al. (2019, see their Table 1) to study the nature of the shell-like overdensity are young star clusters, to which we added two other young star clusters (B111, H86.197). Note that the ages compiled by them come from a variety of sources, while our age estimates were obtained from SMASH data sets and the procedures described in Sections 2 and 3, namely: the decontamination of field stars and the compar-
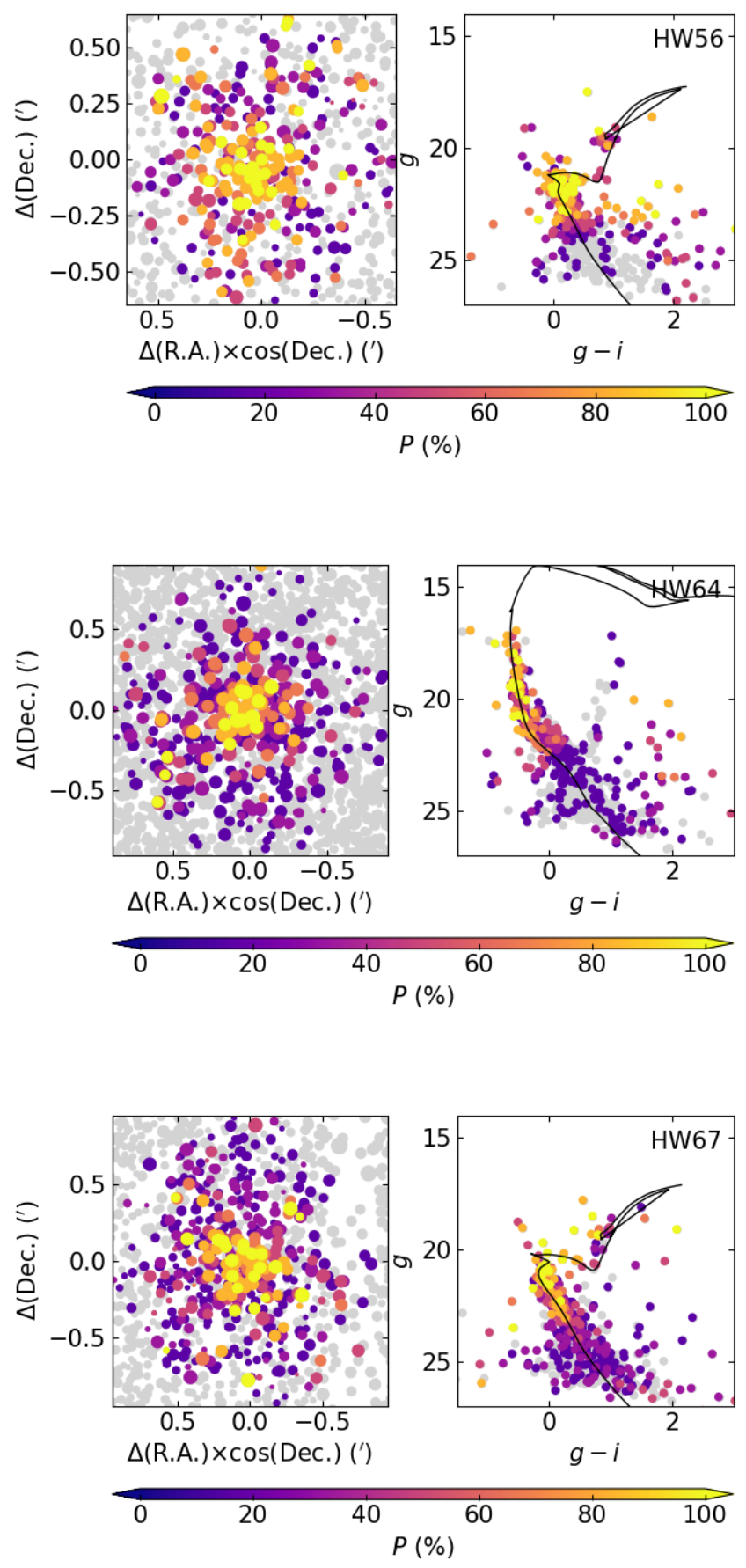

Figure 2. continued.

ison between observed and synthetic CMDs. For these reasons, we think that our derived star cluster properties are homogeneous and more accurate. They come from deeper photometric data; they were obtaining without assuming a mean distance modulus for all the star clusters, and the associated synthetic CMDs best resemble the observed ones among a large number of generated CMDs covering the parameter space. 

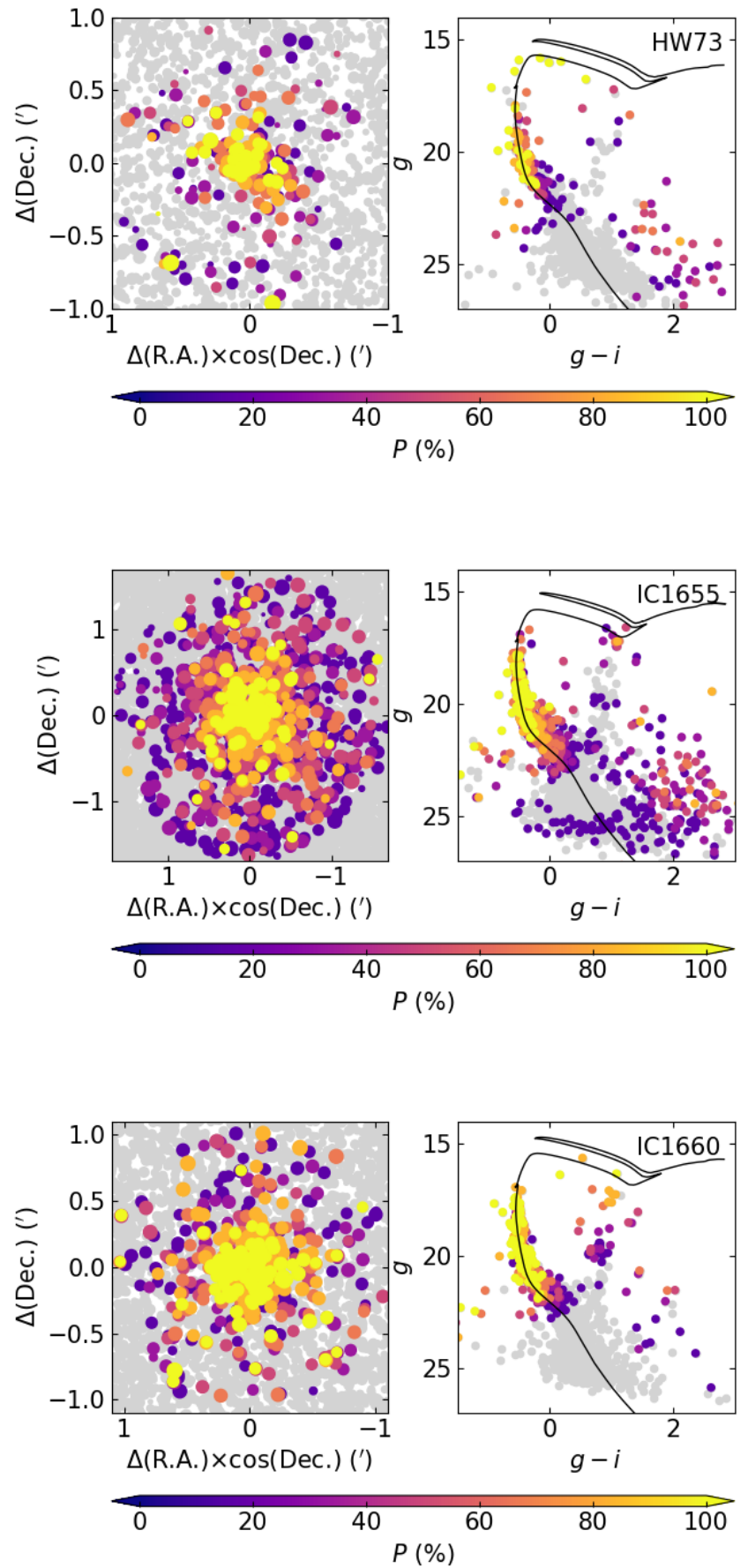

Figure 2. continued.

As Fig. 1 shows, the resulting interstellar reddening values show that the studied clusters are split into two groups with slightly different mean colour excesses $E(B-V)$, that in turn have some particular projected spatial distribution in the sky. It seems that there is a group of mostly outermost star clusters with a mean $E(B-V)$ value of $0.05 \mathrm{mag}$, and another group of star clusters closer to the SMC main body with a mean $E(B-V)$ value of 0.12 mag. Both mean
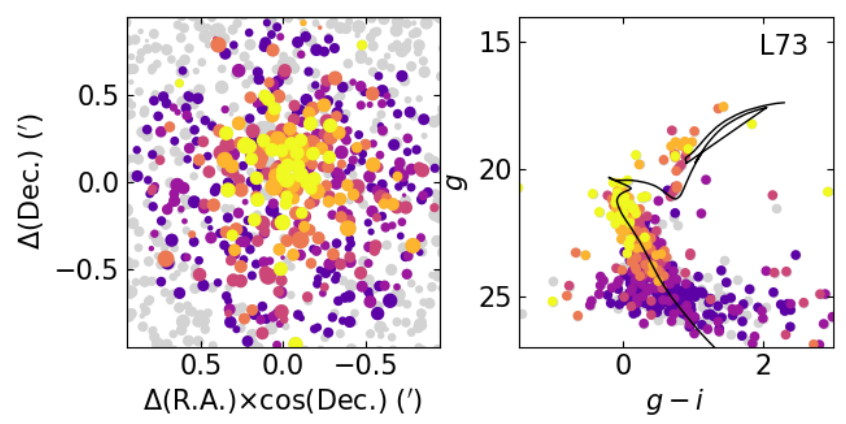

$\begin{array}{ccc}0.5 & 0.0 \quad-0.5 \\ \Delta \text { (R.A. }) \times \cos (\text { Dec. })\left({ }^{\prime}\right)\end{array}$

$0 \quad 20 \quad 40$ $P(\%)$
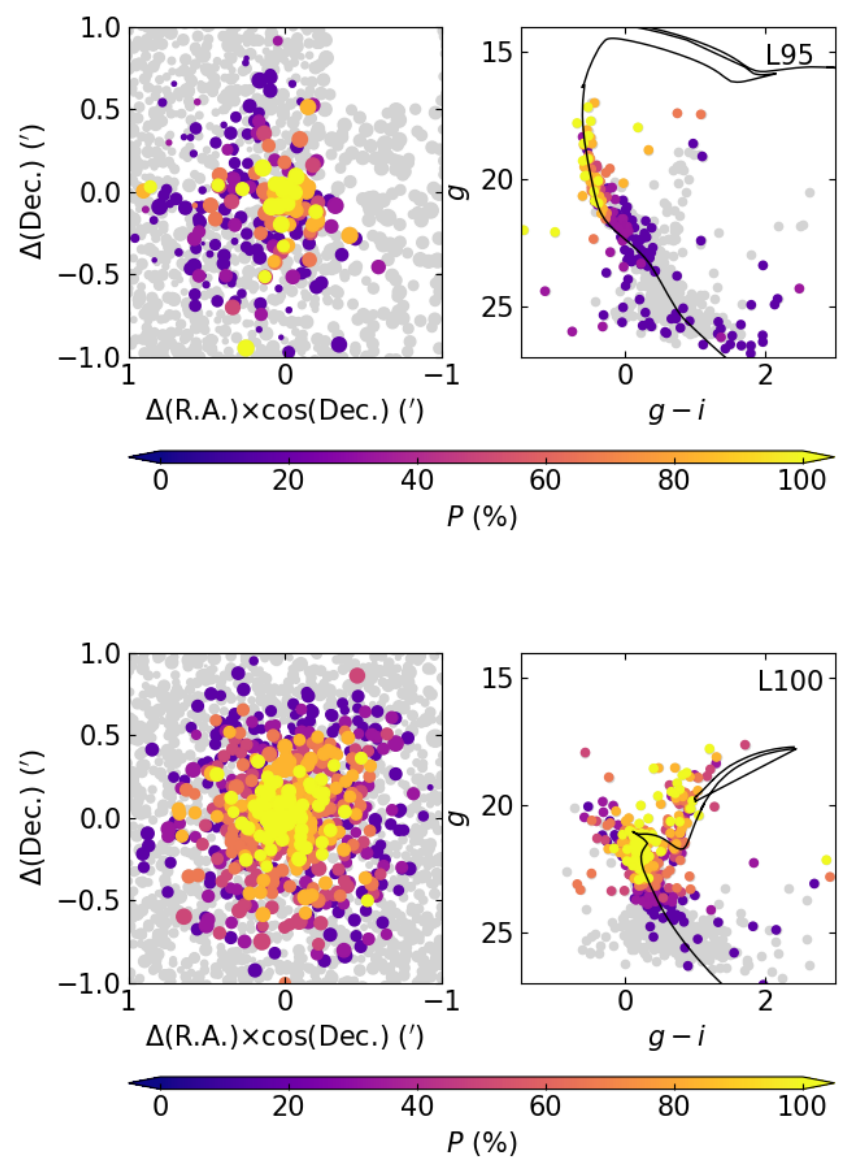

Figure 2. continued.

$E(B-V)$ colour excesses are within the range of values for that part of the SMC according to different SMC reddening maps (see, e.g., Bell et al. 2020; Skowron et al. 2021). With the aim of looking into such a spatial distribution we built Fig. 3, that shows studied older star clusters belonging to the group of objects with relatively small $E(B-V)$ values and most of the younger star clusters being affected by larger interstellar reddening values. Younger star clusters are fre- 


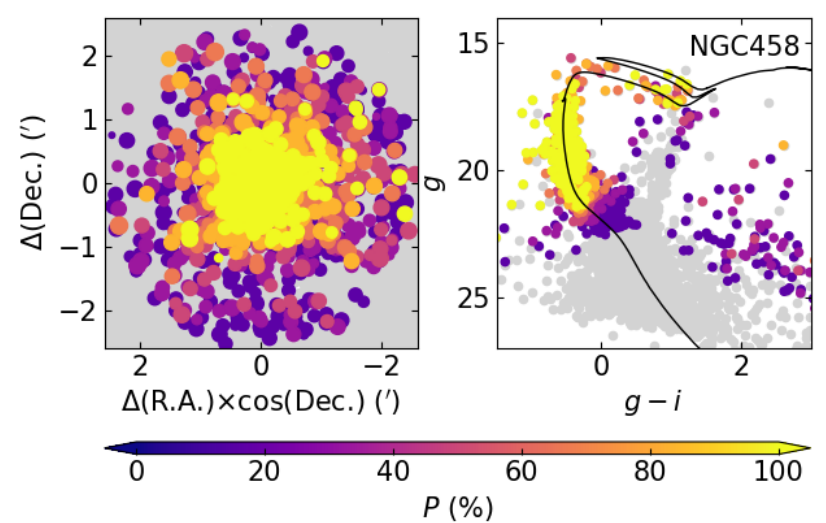

Figure 2. continued.

quently found in regions with gas and dust residuals, so that Figs. 1 and 3 confirm that a region of the shell-like overdensity contains young star clusters and gas/dust residuals (see, also, Martínez-Delgado et al. 2019).

Fig. 3 also reveals a striking distribution of star clusters along the line-of-sight. Older star clusters are located at distances compatible with being part of the SMC main body, whose boundaries are nearly at 56 and $62 \mathrm{kpc}$ from the Sun (Piatti 2021d, and references therein). However, younger star clusters span a wider range of heliocentric distances, from those located in front of the SMC $(D \lesssim 56 \mathrm{kpc})$ up to those placed behind it ( $D \gtrsim 62 \mathrm{kpc})$. Such a range in heliocentric distance is also seen in SMC stellar structures that formed from the interaction with the LMC. For instance, the Magellanic Bridge is located at $D<55 \mathrm{kpc}$ (Wagner-Kaiser \& Sarajedini 2017; Jacyszyn-Dobrzeniecka et al. 2020); the Counter-Bridge, placed towards the northeastern outskirts of the SMC, is at $D>65 \mathrm{kpc}$ (Dias et al. 2021), and the West halo, in the south-west SMC periphery, is at $52 \lesssim D$ $(\mathrm{kpc}) \lesssim 70$ (Dias et al. 2016).

We transformed star cluster equatorial coordinates and heliocentric distances to $3 \mathrm{D}$ cartesian coordinates following the formalism described in (Weinberg \& Nikolaev 2001; van der Marel \& Cioni 2001), and the SMC optical centre coordinates R.A. $=13.1875^{\circ}$, Dec. $=-72.8286^{\circ}$ (Crowl et al. 2001), and its heliocentric distance of $59.0 \mathrm{kpc}$ (Piatti 2021d). Fig. 4 depicts the resulting $X, Y, Z$ coordinates relative to the SMC centre. The $Z$ direction is perpendicular to the $(X, Y)$ plane and is oriented along the line-of-sight to the SMC centre; it increases outwards. The $(X, Y)$ plane, which is a fairly close representation of the distribution of the star clusters seen in the sky (see Fig. 1), shows that the older star clusters are scattered across the shell-like overdensity. When looking at their distribution along the $\mathrm{Z}$ axis, we found that all of them belong to the SMC main body. There are also other seven younger star clusters pertaining to the galaxy that are mingled with the older star clusters. From this finding, we conclude that the shell-like overdensity could not purely be originated by a recent star formation event. These younger star clusters could formed in that part of the galaxy when the studied older star clusters also populated that outermost SMC region.

The possible formation scenario of the younger star clusters can be unveiled by including those star clusters located far away the SMC main body. There are eight young star clusters projected towards the southeast region of the shelllike overdensity, four of them placed nearly $13.0 \mathrm{kpc}$ far away from those in the SMC. Fig. 5 shows the 3D spatial distribution of the studied younger star clusters. It reveals that, for the southeastern group of young star clusters, the younger the star cluster, the larger its $Z$ coordinate. This range of $Z$ values resembles that of tidally perturber/formed SMC stellar structures mentioned above. Additionally, the age gradient seen along the $Z$ direction among the group of southeastern young star clusters tells us about a possible cluster formation wave that started nearly $200 \mathrm{Myr}$ ago (the age of B111) at the outermost region of the SMC and continued outwards until very recently, $\sim 30 \mathrm{Myr}$ ago (the age of HW 64). In order to that cluster formation sequence took place in space and time, gas from the SMC should have stripped off its body, so that while gas is moving outwards, star clusters formed. Here we think that the interaction with the LMC/MW (Besla et al. 2007, 2012) could be responsible for the shell-like overdensity is another possible tidally perturbed/formed SMC stellar structure. This outcomes bring additional support to previous speculations and results of this possible formation mechanism (Glatt et al. 2010; Skowron et al. 2014; Jacyszyn-Dobrzeniecka et al. 2017; Ripepi et al. 2017; Zivick et al. 2018b,a, and references therein).

Fig. 6 shows a slight correlation between the metallicity and the relative depth along the line-of-sight to the SMC centre of the eight young star clusters in the southeastern region of the shell-like overdensity. Such a trend could be interpreted as a chemical abundance enrichment process with time along the $Z$ direction. Nevertheless, further investigations from more accurate metallicities are needed. It is also worth mentioning that among these eight young star clusters the more massive (total present mass $\sim 4500 \mathrm{M}_{\odot}$ ) ones formed in the SMC main body, while those less massive $(<$ $\left.2000 \mathrm{M}_{\odot}\right)$ are located farther away from the SMC. As far as the age-metallicity relationship of SMC star clusters is considered, Fig. 7 shows that the studied star clusters follow the known trend and dispersion derived from observed star clusters (see Piatti \& Geisler 2013), field stars (Harris \& Zaritsky 2004), and theoretical models (Pagel \& Tautvaisiene 1998), respectively.

\section{CONCLUSIONS}

We studied twenty catalogued star clusters projected onto the so-called shell-like overdensity, a stellar structure located in the outer northeastern region of the SMC, with the aim of estimating accurate star cluster properties. We embarked on this investigation motivated by the fact the star clusters analysed by Martínez-Delgado et al. (2019) were assumed to be at the same mean SMC distance, while the galaxy is known to be more extended in depth than its projection in the plane of the sky. An additional reason supporting this study is the need of decontamination of field stars from the star cluster CMDs in order to derive accurate astrophysical 

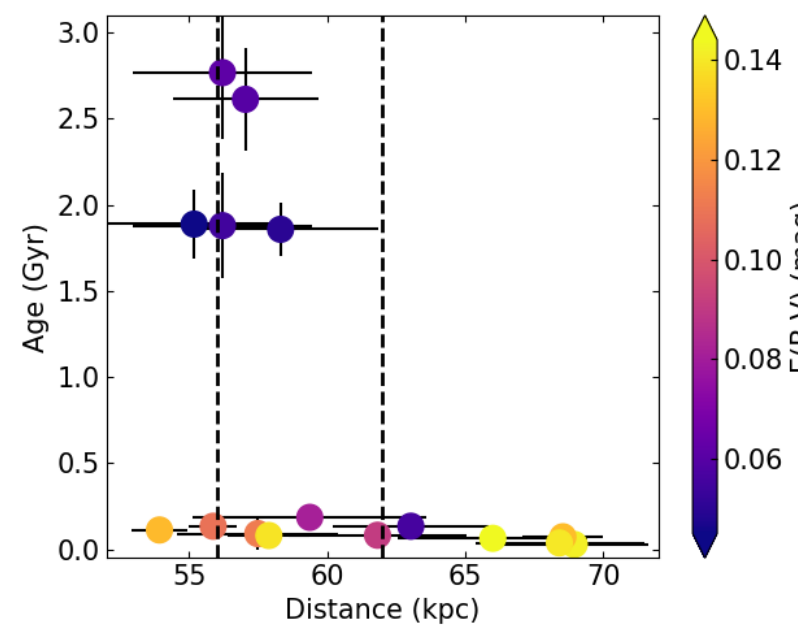

$-0.12$

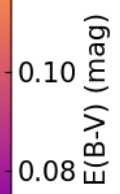

0.06

Figure 3. Relation between different star cluster properties. The vertical dashed lines represent the boundaries of the SMC main body along its line-of-sight (Piatti 2021d).

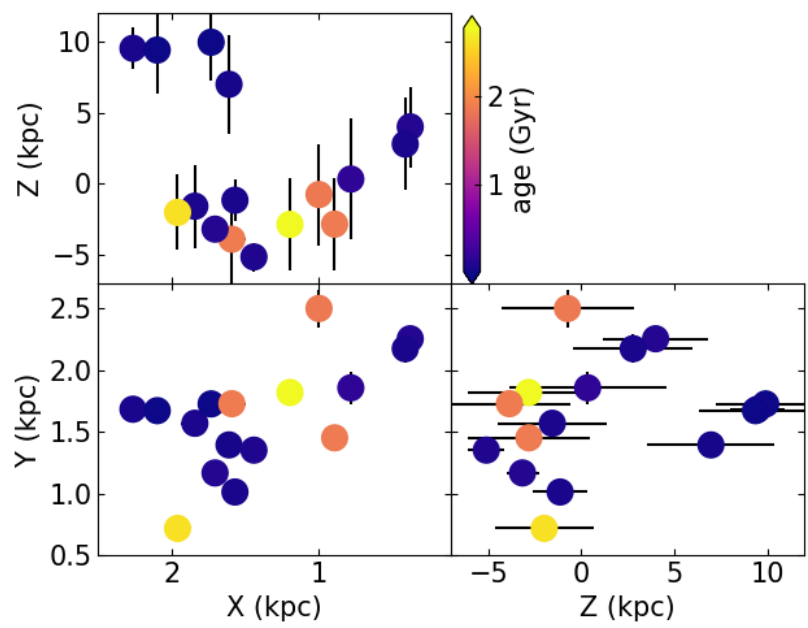

Figure 4. 3D spatial distribution of SMC star clusters in the shelllike overdensity. Errobars are included; for $X$ and $Y$ coordinates they are smaller than the size of the symbols.

parameters. Ages and metallicities were previously derived for some of these star clusters from different sources, so that an effort of determining them homogeneously is also valuable.

A robust field star cleaning procedure was applied to the entire star cluster sample using SMASH data sets, which unveiled that four objects are not genuine star clusters (OGLESMC 274, 275, 276, and 277), while the remaining true star clusters are split in two age groups: a younger group (30$200 \mathrm{Myr})$ with eleven star clusters, and an older one (1.7-2.8 Gyr) composed of five star clusters. We confirmed the youth of the nive star clusters used by Martínez-Delgado et al. (2019). When star cluster distances are examined, we found that the older star clusters and seven young clusters pertain to the SMC main body, while four young clusters are nearly $13.0 \mathrm{kpc}$ farther away. This outcome suggests a more extended shell-like overdensity structure along the line-of-

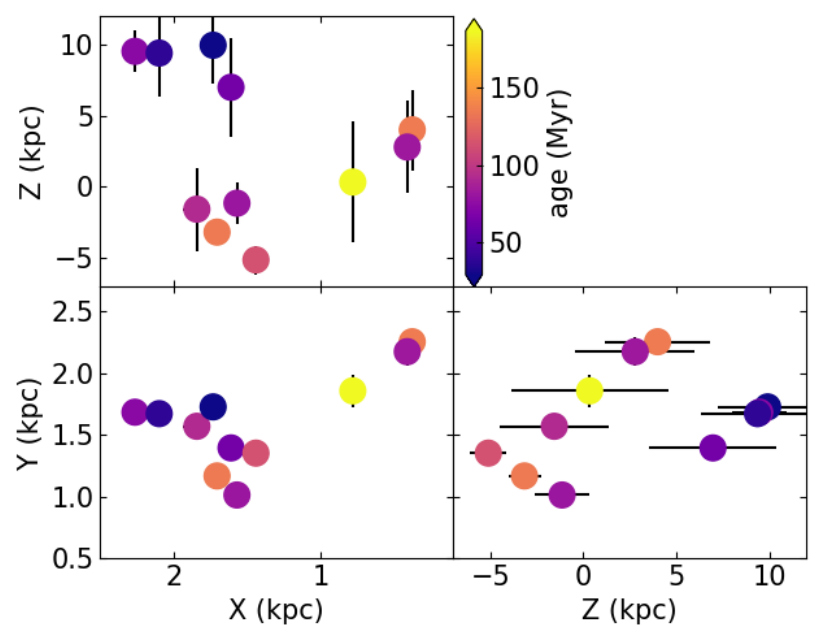

Figure 5. Same as Fig. 4 for younger star clusters.

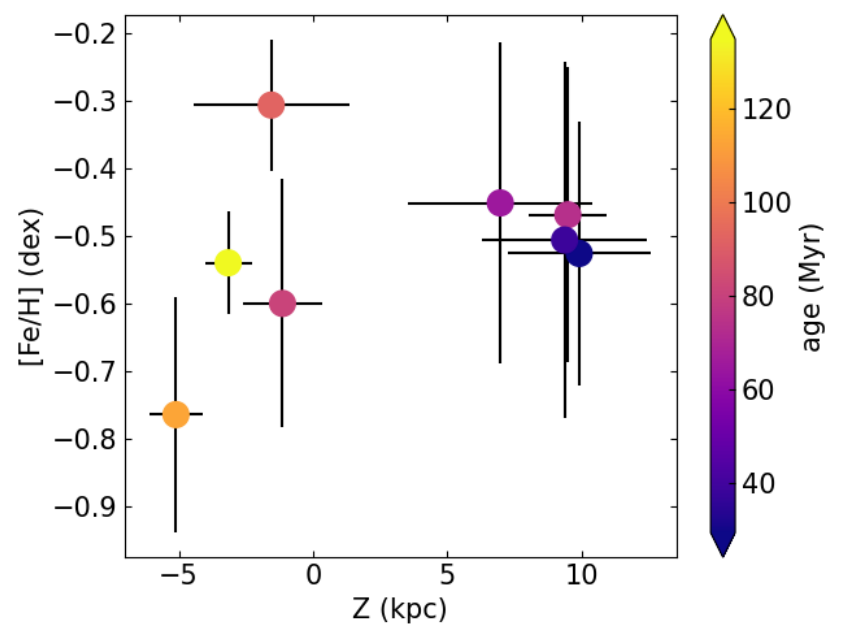

Figure 6. Relation between the metallicity and the relative depth from the SMC centre of the group of younger star clusters located in the southeastern region of the shell-like overdensity.

sight than in the plane of the sky. The younger star clusters that span a wide range of depth values are located in the southeastern region of the shell-like overdensity. Curiously, they are affected by slightly larger colour excesses as compared with the other star clusters in the shell-like overdensity. There is also a correlation between their ages and their distances along the lne-of.sight to the SMC centre, in the sense that younger clusters $(\sim 30 \mathrm{Myr})$ are farther than those relatively older $(\sim 200 \mathrm{Myr})$. A less clear correlation with metallicity also arises, with more metal-rich young star clusters placed farther from the SMC.

These observational evidence point to the possibility that the younger star clusters in the shell-like overdensity are part of a tidally perturbed/formed SMC stellar structure from gas striped off its body, caused by the interaction with the LMC/MW. At the moment of that interaction intermediate-age star clusters populated the shell-like overdensity region, while star clusters formed out of gas clouds pulled out by the interacting galaxy. The age-metallicity re- 


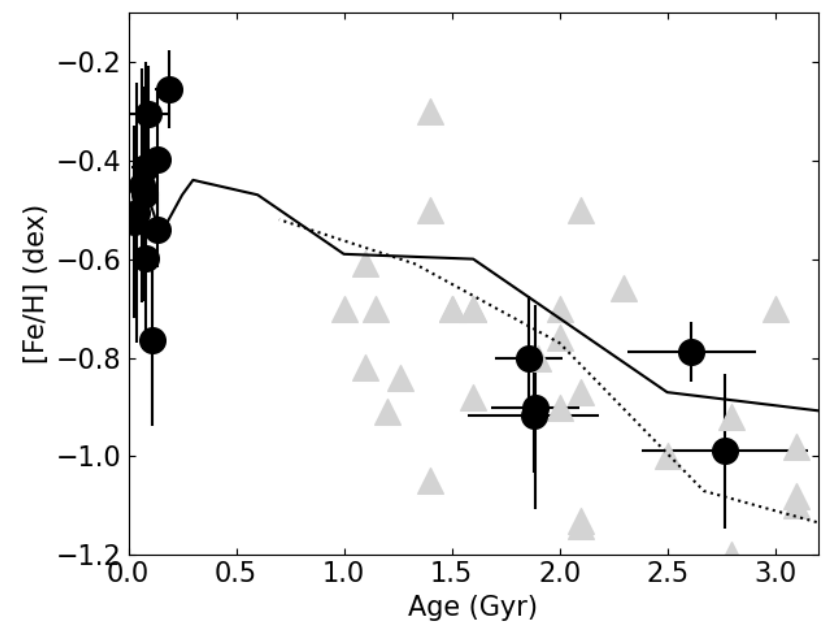

Figure 7. The age-metallicity relationship of shell-like overdensity star clusters. Triangles represent the SMC star clusters from Piatti \& Geisler (2013), while solid and dotted lines correspond to the age-metallicity relationships derived from field stars (Harris \& Zaritsky 2004), and theoretical models (?), respectively.

lationship of the studied star clusters are in agreement with our previous knowledge of age-metallicity relationships built from observed star clusters, star fields, and theoretical models.

The present results can stimulate further deep observational campaigns of SMC star clusters. Indeed, we used Gaia EDR3 $^{2}$ data sets to performe the analysis described in Section 2, by extracting parallaxes $(\varpi)$, proper motions in right ascension (pmra) and declination (pmdec), excess noise (epsi), the significance of excess of noise (sepsi), and $G, B P$, and $R P$ magnitudes for stars located within a radius $9^{\prime}$ from the respective cluster centres. We limited our sample to stars with proper motion errors $\leq 0.1 \mathrm{mas} / \mathrm{yr}$, following the suggestion by Piatti (2021d, and reference therein), who found that the larger the individual proper motion errors of SMC stars, the larger the derived errors of the mean SMC cluster proper motions. We minimized the presence of foreground stars by favoring distant stars (i.e., $|\varpi| / \sigma(\varpi)<3$ ) and pruned the data with sepsi $<2$ and epsi $<1$, which define a good balance between data quality and the number of retained objects for our sample (see also Ripepi et al. 2019). Once we applied the cleaning procedure to the Gaia EDR3 data sets, some star clusters were left with few or no stars, because of not enough photometric depth.

\section{DATA AVAILABILITY}

Data used in this work are publicly available at https://datalab.noao.edu/smash/smash.php site.

\section{ACKNOWLEDGEMENTS}

I thank the referee for the thorough reading of the manuscript and suggestions to improve it.

2 https://archives.esac.esa.int/gaia.
This research uses services or data provided by the Astro Data Lab at NSF's National Optical-Infrared Astronomy Research Laboratory. NSF's OIR Lab is operated by the Association of Universities for Research in Astronomy (AURA), Inc. under a cooperative agreement with the $\mathrm{Na}-$ tional Science Foundation.

This work has made use of data from the European Space Agency (ESA) mission Gaia (https://www.cosmos. esa.int/gaia), processed by the Gaia Data Processing and Analysis Consortium (DPAC, https://www.cosmos.esa. int/web/gaia/dpac/consortium). Funding for the DPAC has been provided by national institutions, in particular the institutions participating in the Gaia Multilateral Agreement.

\section{REFERENCES}

Bell C. P. M., et al., 2020, MNRAS, 499, 993

Besla G., Kallivayalil N., Hernquist L., Robertson B., Cox T. J., van der Marel R. P., Alcock C., 2007, ApJ, 668, 949

Besla G., Kallivayalil N., Hernquist L., van der Marel R. P., Cox T. J., Kereš D., 2012, MNRAS, 421, 2109

Bica E., Westera P., Kerber L. d. O., Dias B., Maia F., Santos João F. C. J., Barbuy B., Oliveira R. A. P., 2020, AJ, 159, 82

Bressan A., Marigo P., Girardi L., Salasnich B., Dal Cero C., Rubele S., Nanni A., 2012, MNRAS, 427, 127

Crowl H. H., Sarajedini A., Piatti A. E., Geisler D., Bica E., Clariá J. J., Santos Jr. J. F. C., 2001, AJ, 122, 220

Dias B., Kerber L., Barbuy B., Bica E., Ortolani S., 2016, A\&A, 591, A11

Dias W. S., Monteiro H., Moitinho A., Lépine J. R. D., Carraro G., Paunzen E., Alessi B., Villela L., 2021, MNRAS, 504, 356

Efron B., 1982, The Jackknife, the Bootstrap and other resampling plans

Gaia Collaboration et al., 2016, A\&A, 595, A1

Gaia Collaboration et al., 2018, A\&A, 616, A1

Glatt K., et al., 2008, AJ, 136, 1703

Glatt K., Grebel E. K., Koch A., 2010, A\&A, 517, A50

Graczyk D., et al., 2020, arXiv e-prints, p. arXiv:2010.08754

Harris J., Zaritsky D., 2004, AJ, 127, 1531

Jacyszyn-Dobrzeniecka A. M., et al., 2017, Acta Astron., 67, 1

Jacyszyn-Dobrzeniecka A. M., et al., 2020, ApJ, 889, 26

Knuth K. H., 2018, optBINS: Optimal Binning for histograms (ascl:1803.013)

Kroupa P., 2002, Science, 295, 82

Martínez-Delgado D., et al., 2019, A\&A, 631, A98

Martínez-Vázquez C. E., Salinas R., Vivas A. K., 2021, AJ, 161, 120

Muraveva T., et al., 2018, MNRAS, 473, 3131

Nidever D. L., et al., 2017, AJ, 154, 199

Nidever D. L., et al., 2021, AJ, 161, 74

Pagel B. E. J., Tautvaisiene G., 1998, MNRAS, 299, 535

Perren G. I., Vázquez R. A., Piatti A. E., 2015, A\&A, 576, A6

Piatti A. E., 2017, ApJ, 834, L14

Piatti A. E., 2018, MNRAS, 477, 2164

Piatti A. E., 2021a, AJ, 161, 199

Piatti A. E., 2021b, A\&A, 647, A11

Piatti A. E., 2021c, A\&A, 647, A47

Piatti A. E., 2021d, A\&A, 650, A52

Piatti A. E., Bica E., 2012, MNRAS, 425, 3085

Piatti A. E., Geisler D., 2013, AJ, 145, 17

Ripepi V., et al., 2017, MNRAS, 472, 808

Ripepi V., Molinaro R., Musella I., Marconi M., Leccia S., Eyer L., 2019, A\&A, 625, A14

Skowron D. M., et al., 2014, ApJ, 795, 108 


\section{Andrés E. Piatti}

Skowron D. M., et al., 2021, ApJS, 252, 23

Tremmel M., et al., 2013, ApJ, 766, 19

Wagner-Kaiser R., Sarajedini A., 2017, MNRAS, 466, 4138

Weinberg M. D., Nikolaev S., 2001, ApJ, 548, 712

Zivick P., et al., 2018a, arXiv e-prints

Zivick P., et al., 2018b, ApJ, 864, 55

van der Marel R. P., Cioni M.-R. L., 2001, AJ, 122, 1807

von Hippel T., 2005, ApJ, 622, 565

This paper has been typeset from a $\mathrm{T}_{\mathrm{E}} \mathrm{X} / \mathrm{LATEX}$ file prepared by the author. 